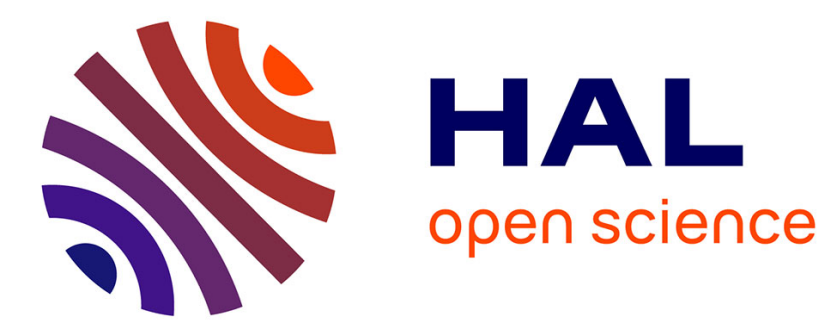

\title{
Te-As-Se glass destabilization using high energy milling
}

Laurent Calvez, Enora Lavanant, Anna Novikova, Claudia Gonçalves, Bruno

Bureau, Virginie Nazabal, Thierry Jouan, Xiang-Hua Zhang

\section{To cite this version:}

Laurent Calvez, Enora Lavanant, Anna Novikova, Claudia Gonçalves, Bruno Bureau, et al.. Te-As-Se glass destabilization using high energy milling. Journal of Non-Crystalline Solids, 2018, 480, pp.28-33. 10.1016/j.jnoncrysol.2017.08.017 . hal-01660838

\section{HAL Id: hal-01660838}

\section{https://hal-univ-rennes1.archives-ouvertes.fr/hal-01660838}

Submitted on 11 Dec 2017

HAL is a multi-disciplinary open access archive for the deposit and dissemination of scientific research documents, whether they are published or not. The documents may come from teaching and research institutions in France or abroad, or from public or private research centers.
L'archive ouverte pluridisciplinaire HAL, est destinée au dépôt et à la diffusion de documents scientifiques de niveau recherche, publiés ou non, émanant des établissements d'enseignement et de recherche français ou étrangers, des laboratoires publics ou privés. 


\title{
Te-As-Se glass destabilization using high energy milling
}

\author{
Laurent Calvez $z^{1, *}$, Enora Lavanant ${ }^{1}$, Anna Novikova ${ }^{1}$, Claudia Goncalves ${ }^{1}$, Bruno Bureau ${ }^{1}$ \\ Virginie Nazabal, Thierry Jouan ${ }^{1}$, Xiang-Hua Zhang ${ }^{1}$ \\ ${ }^{1}$ Equipe Verres et Céramiques, UMR-CNRS 6226 Institut des Sciences Chimiques de Rennes 1, \\ Université de Rennes 1, 263 Avenue Général Leclerc, Rennes, 35042, France \\ * laurent.calvez@univ-rennes1.fr
} $+33-223236713$

\begin{abstract}
The $\mathrm{Te}_{20} \mathrm{As}_{30} \mathrm{Se}_{50}$ (TAS) glass has been studied under the extreme conditions of high energy milling. Starting from the raw materials, an amorphization process occurs progressively reaching an unstable intermediate state and the final stable state then. Despite its high resistance against crystallization when made using silica tubes, an intermediate state is reached when mechanically alloyed into powder. The evolution of the glass structure has been investigated using Raman spectroscopy and MAS NMR of ${ }^{77} \mathrm{Se}$ according to the milling time. Optimized parameters and conditions to compact milled powders by Spark Plasma Sintering show the possibility to design infrared windows transparent from 2 to $20 \mu \mathrm{m}$. A correlation to the thermo-mechanical properties of densified powder using hot pressing has been made.
\end{abstract}

Keywords : glasses, chalcogenide, mechanical alloying, sintering, Spark Plasma Sintering

\section{Introduction}

Night Vision is the first thing that comes to mind when speaking of chalcogenide glasses capable of transmitting light in the infrared [1-2]. But these glasses have other advantages; they are also very good candidate for chemical species sensors since most of the chemical or biochemical molecules present fundamental vibrations in the mid-infrared range, having a specific and recognizable signature. The optical fiber of chalcogenide glass can thus be used for the detection of chemical species in the liquid or gaseous state. Indeed, the use of this type of glass as a chemical sensor has already been demonstrated [3-4]. It is thus possible to produce biosensors from chalcogenide fibers such as $\mathrm{Te}_{20} \mathrm{As}_{30} \mathrm{Se}_{50}$ (TAS) glass composition that enable faster and less invasive diagnostics for infectious diseases, chronic, and cancer [5]. Their efficiency can then be monitored by tapering the fiber diameter (about $100 \mu \mathrm{m}$ ). This step occurs after the fiber drawing on section of about $4 \mathrm{~cm}$ length that are heated again. This multistep process is very time consuming to obtain reproducible section diameters of sensors.

The objective of this work is therefore to develop a way to easily and rapidly shape the $\mathrm{Te}_{20} \mathrm{As}_{30} \mathrm{Se}_{50}$ (TAS) glass into bulks or tapers. Thus, the possibility to obtain TAS amorphous powder by mechanical alloying, cheaper method than conventional melt-quenching method has been investigated. The possibility to mix and get amorphous alloys is commonly used to obtain metallic glasses [6-7]. This process has already been investigated in germanium based chalcogenide glasses [8-9]. Then, coupled to the mechanical synthesis, flash sintering using Spark Plasma Sintering (SPS) will be used to compact and mold the glass powder.

\section{Experimental details}


The synthesis of amorphous powders was carried out in a planetary mill (Retsch® PM 100), using a bowl $(125 \mathrm{ml})$ and 6 balls (diameter $20 \mathrm{~mm}$ ) in tungsten carbide from raw metals (Te, As and $\mathrm{Se}, 5 \mathrm{~N})$. Syntheses were performed under controlled atmosphere of argon in a glove box.

Furthermore, in order to avoid overheating of the system, a grinding cycle was imposed, alternating rotation for 3 minutes in a sense, a break of 3 minutes and a rotation for 3 minutes in the opposite direction. It may be noted in addition that the milling was regularly stopped in order to collect powder samples to monitor the structural evolution thereof.

In parallel, some TAS bulk glasses were synthesized using the process described by Zhang et al. [10]. Some samples were then grinded for 30 minutes in the planetary grinder, in order to compare the properties and structure reached in both methods.

Thus, samples have been defined as TAS-MM-Xh for mechanical milled powder, $\mathrm{X}$ being the time of milling and TAS-G for the base glass being grinded for 30minutes. The reference will be the TAS glass made by melt quenching that will be called TAS base glass.

Thermo-mechanical analysis were performed using Differential scanning calorimetry (DSC) (DSC Q20 TA Instruments) with a temperature ramp of $10^{\circ} \mathrm{C} / \mathrm{min}$ and Vickers hardness measurements using a Matsuzawa microindentor (100g, 5s), average of 10 measures.

Structural analysis of amorphous powder has been investigated using XRD (Phillips PW3710 diffractometer, NMR of the ${ }^{77} \mathrm{Se}$ atom and Raman spectroscopy for different times of grinding.

Raman acquisitions are made with the HR800- $785 \mathrm{~nm}$ optical density D2 with a laser at? nm. Studies were conducted on green pellets of powders taken after $100 \mathrm{~h}$ and $160 \mathrm{~h}$ of grinding and on a bulk sample compacted by SPS on powder of $160 \mathrm{~h}$ of milling.

All NMR spectra 77Se $(S=1 / 2)$ were recorded at room temperature on the Avance 300 Bruker spectrometer operating at $57.28 \mathrm{MHz}$ using a $4 \mathrm{~mm}$ rotor turned under magic angle of $54.74^{\circ}$ (MAS, Magic Angle Spinning) at $14 \mathrm{kHz}$.

\section{Results}

The $\mathrm{Te}_{20} \mathrm{As}_{30} \mathrm{Se}_{50}$ glass presents a high stability against crystallization having a $\mathrm{Tg}$ of $135^{\circ} \mathrm{C}\left( \pm 2^{\circ} \mathrm{C}\right)$ [11], showing no crystallization peak. This high stability allows its easy drawing into fibers of specific design with a high flexibility. The thermal properties of TAS powder obtained after different milling duration are listed in Table 3.

Table 1. Evolution of the characteristic temperatures of the TAS powder as a function of the milling time.

\begin{tabular}{l|lll}
\hline Milling time & $\mathrm{T}_{\mathrm{g}}\left({ }^{\circ} \mathrm{C}\right)\left( \pm 2{ }^{\circ} \mathrm{C}\right)$ & $\mathrm{T}_{\mathrm{x}}\left({ }^{\circ} \mathrm{C}\right)\left( \pm 2{ }^{\circ} \mathrm{C}\right)$ & $\Delta \mathrm{T}\left(\mathrm{T}_{\mathrm{x}}-\mathrm{T}_{\mathrm{g}}\right)$ \\
\hline $1 \mathrm{~h}$ & 47 (pure Se) & 81 & 34 \\
$2 \mathrm{~h}$ & 54 & 82 & 28 \\
$3 \mathrm{~h}$ & 62 & 82 & 20 \\
$5 \mathrm{~h}$ & 70 & 90 & 20 \\
\hline
\end{tabular}




\begin{tabular}{l|lll}
\hline $10 \mathrm{~h}$ & 107 & 166 & 59 \\
$15 \mathrm{~h}$ & 125 & 202 & 77 \\
$20 \mathrm{~h}$ & 130 & 215 & 85 \\
$40 \mathrm{~h}$ & 132 & 217 & 85 \\
$60 \mathrm{~h}$ & 133 & 223 & 90 \\
$80 \mathrm{~h}$ & 135 & 226 & 91 \\
$100 \mathrm{~h}$ & 135 & 227 & 92 \\
$140 \mathrm{~h}$ & 135 & - & - \\
$160 \mathrm{~h}$ & 135 & - & - \\
\hline
\end{tabular}

During the first two hours of grinding, the thermal answer corresponds to pure amorphous Se. The gradual increase of $\mathrm{Tg}$ shows the progressive reaction of selenium, arsenic and tellurium to lead to the formation of the amorphous phase of the TAS after about $40 \mathrm{~h}$.

While the theoretical Tg of the TAS glass can be rapidly reached, a residual crystallization peak can be observed at about $225^{\circ} \mathrm{C}$. With longer milling time, the $\mathrm{Tg}$ remains stable and the peak of crystallization shifts to higher temperatures until it disappears after140 hours.

Thereafter, the mechanical alloying could be stopped after 60 hours of milling to compact and shape the glass as the $\mathrm{Tg}$ reached a plateau and the difference between $\mathrm{Tx}$ and $\mathrm{Tg}$ is high enough to ensure a compaction without crystallization by SPS. In fact, SPS is known as a fast and low temperature way to compact pellets into their final shape. Sintering is usually performed at a temperature close to Tg for several minutes.

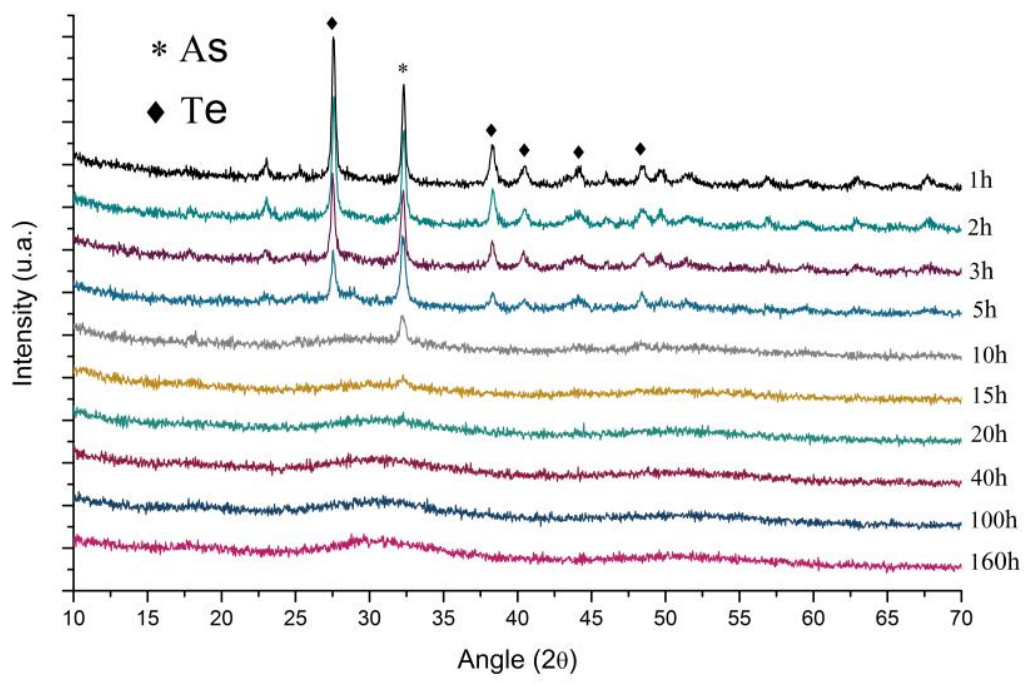


Figure 1. Diffractograms of powders obtained after different grinding time by mechanical synthesis.

The diffractograms showed no peak corresponding to crystalline selenium in the first hours of grinding demonstrating its rapid amorphization. After 5 hours of milling part of the tellurium and arsenic remains in their crystalline form. After 10 hours of milling, tellurium is completely amorphous while crystalline arsenic disappears after $40 \mathrm{~h}$ of milling leading to a fully amorphous powder. It is important to note that no crystalline phase is generated during milling.

In order to understand the difficulty to stabilize the glassy matrix, crystallization experiments were performed on powder milled for 100h, still presenting a crystallization peak in DSC curves.

Powders were heat treated at $\mathrm{Tx}\left(\mathrm{Tg}+90^{\circ} \mathrm{C}\right)$ for 1 and $3 \mathrm{~h}$ in a ventilated furnace.

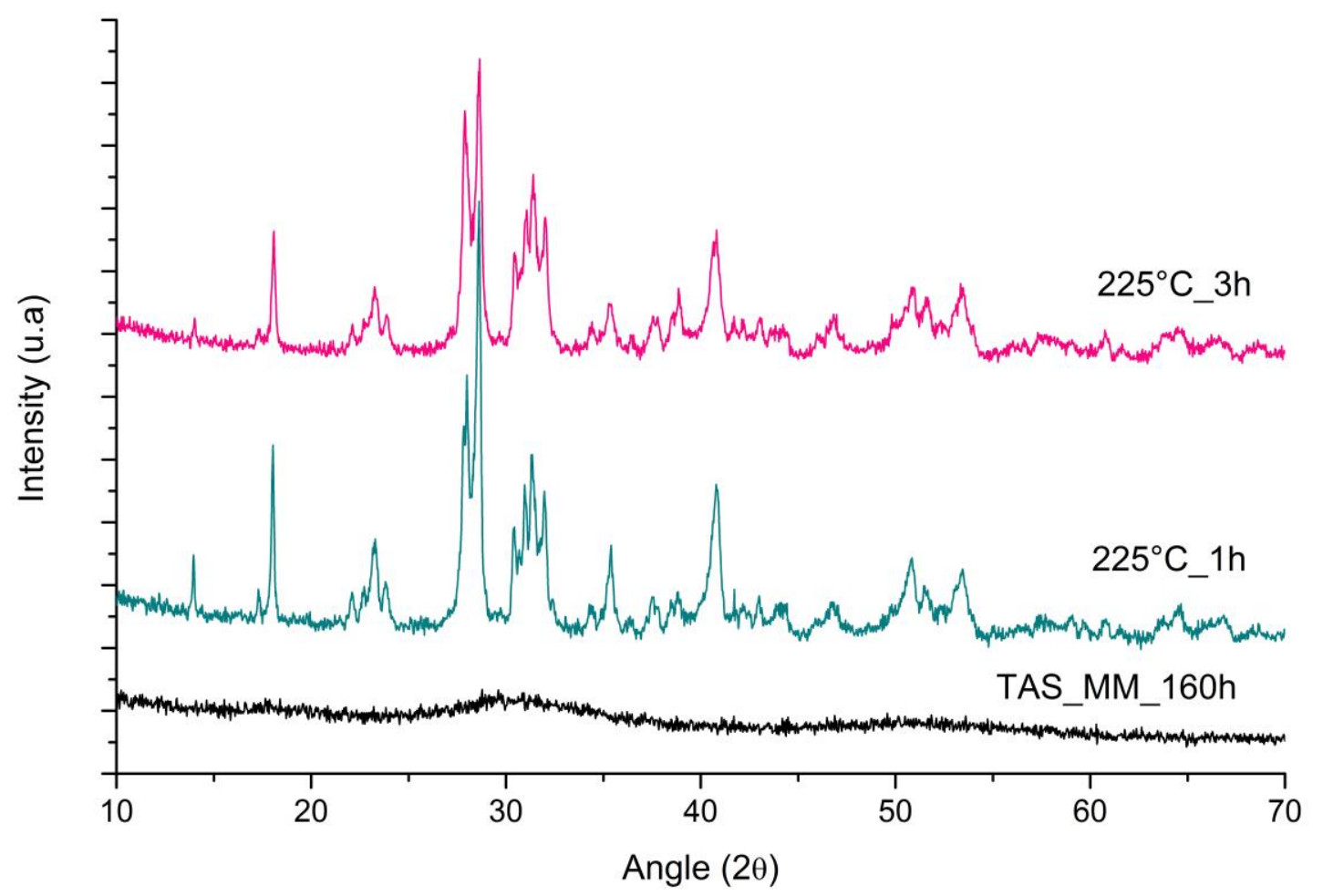

Figure 2. XRD of $100 \mathrm{~h}$ milled powder heat treated for different duration at $\mathrm{Tg}+90^{\circ} \mathrm{C}$ meaning $225^{\circ} \mathrm{C}$.

As shown in figure 2, $\mathrm{As}_{2} \mathrm{Se}_{3}$ crystals are quickly produced within the glassy matrix. This phase remains unchanged after $3 \mathrm{~h}$ of ceramization. This experiment clearly demonstrates the first interaction between Se and As which is favored compared to Te. One can assume that the high specific surface favors a fast and not homogeneous surfacic crystallization process.

\section{Molding process}


The first SPS sintering tests were conducted on molds made of graphite of $10 \mathrm{~mm}$ inner diameter using a sheet of Papyex ${ }^{\circledR}$ (flexible graphite sheet) to ensure the release of the solid as well as protect the diffusion of the graphite component from the mold. Green pellets ( 3 tons) were made to facilitate the shaping and avoid external pollution. Bertrand et al. showed the efficiency of this method to avoid graphite contamination depending on the grain size [12].

First experiments were carried on TAS-MM-160h sintered in graphite molds at two temperatures close to $\operatorname{Tg}\left( \pm 5^{\circ} \mathrm{C}\right)$, results of transmittance are presented in figure 3 .

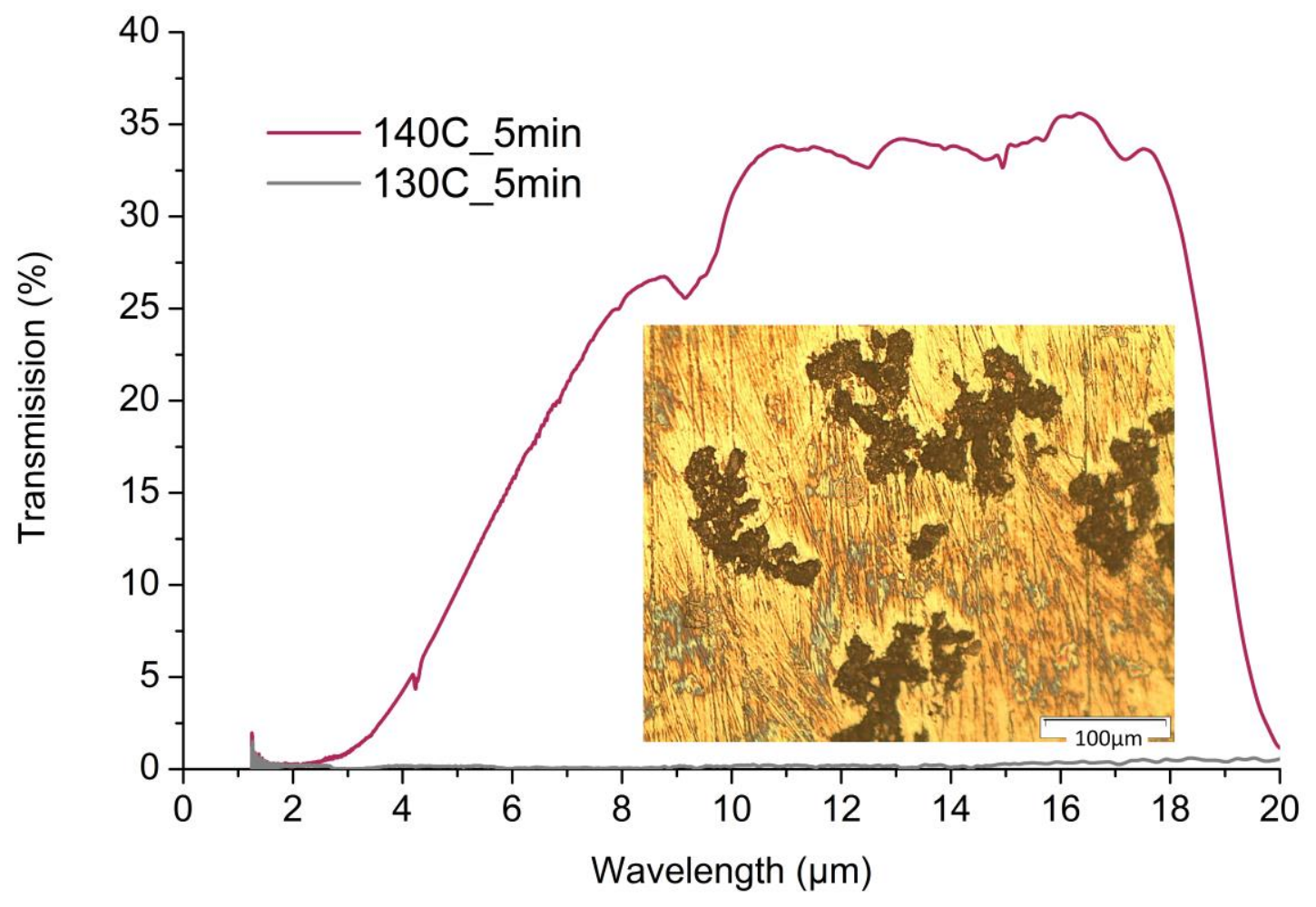

Figure 3. Transmission of sintered TAS-MM-160h after SPS treatment under at $130^{\circ} \mathrm{C}$ and $140^{\circ} \mathrm{C}$ a pressure $50 \mathrm{MPa}$ for 5 minutes. The inset is an image of the glass surface after polishing from optical microscope of the sintered pellet at $140^{\circ} \mathrm{C}$ for $5 \mathrm{~min}$ (scale : $100 \mu \mathrm{m}$ ).

As observable in the figure 3 , temperature appears as a major criterion to control the glass compaction. In fact, $5^{\circ} \mathrm{C}$ below $\mathrm{Tg}$ pellets are not densified enough to transmit. However, samples sintered at $140{ }^{\circ} \mathrm{C}$ present a maximum of $35 \%$, far from the theoretical TAS transmittance of $62 \%$. The densification was calculated from the theoretical glass density $(4,88)$. Even if a densification of $99 \%$ has been determined, the presence of carbon particles of hundreds of microns in the samples may explain the significant MIE scattering, phenomena already observed by Hubert et al. [8].

First, in order to optimize sintering parameters, a purified bulk TAS made by melt-quenching has been grinded for 30 minutes in the planetary grinder. This powder is called TAS-G30. Also, to obtain an effective barrier to carbon diffusion and thereby improve the transmission of the samples, Papyex® foils were replaced by molybdenum or aluminum foils at the top and bottom of the green pellet. The 
low sintering temperatures compared to conventional glasses allow the use of metal foils without diffusion.

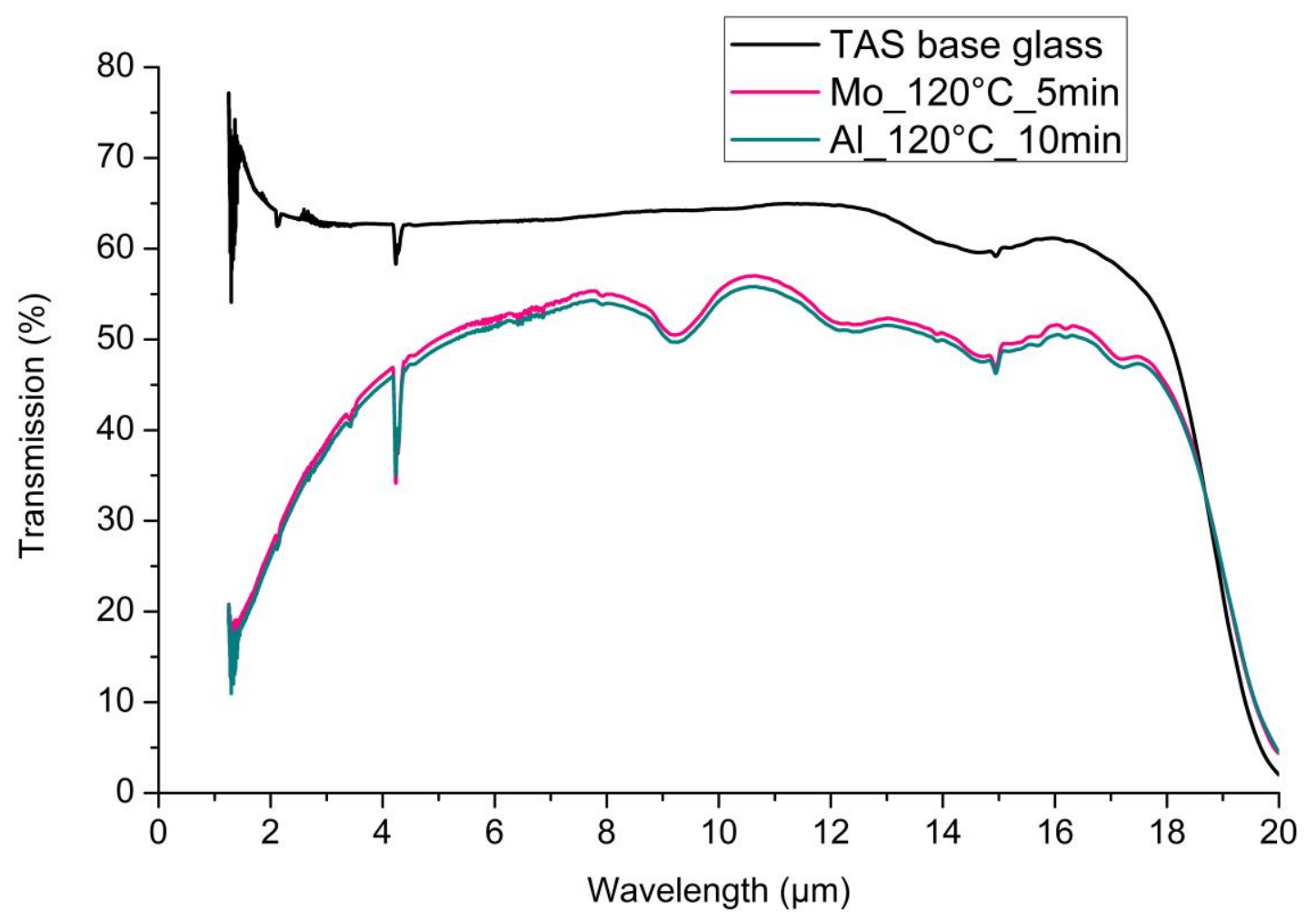

Figure 4. Transmission curve of the TAS base glass and TAS bulk obtained from TAS-G30 powder by SPS sintering.

As observable in Figure 4, the maximum transmission of the TAS glass synthesized by meltquenching is $62 \%$ ( $\mathrm{n}=2.91$ to 10 microns) between 1 and $19 \mu \mathrm{m}$, for a pellet of $1.5 \mathrm{~mm}$ in thickness. Sintered glasses all have broad transparency window in the infrared (2-19 microns). In addition, a maximum transmission of $57 \%$ is obtained for a solid sintered at $120^{\circ} \mathrm{C}$ for 5 minutes using molybdenum or aluminum foils of $0.1 \mathrm{~mm}$. Moreover, slight absorption bands are observable meaning that the glass sintered by SPS contains traces of oxygen and hydrogen. Since the base glass does not show these absorptions, a contamination of the powder occur when preparing the green pellet in atmosphere. Given the small particle size (a few microns), the powder thus has a high specific surface area and therefore is highly responsive, making it very susceptible to corrosion by the atmosphere. Nonetheless, the metal barrier is efficient to decrease scattering losses. 
Densification was calculated from the density of the glass made by conventional melt-quenching route $(\mathrm{d}=4.88 \mathrm{~g} . \mathrm{cm}-3)$, both of the sintered samples present a densification of $99.0 \pm 0.2 \%$. Scatterings can be correlated to the $1 \%$ of densification missing showing the presence of residual porosity. Samples were cleaved to observe the inner part by SEM, shots are combined in Figure 8.
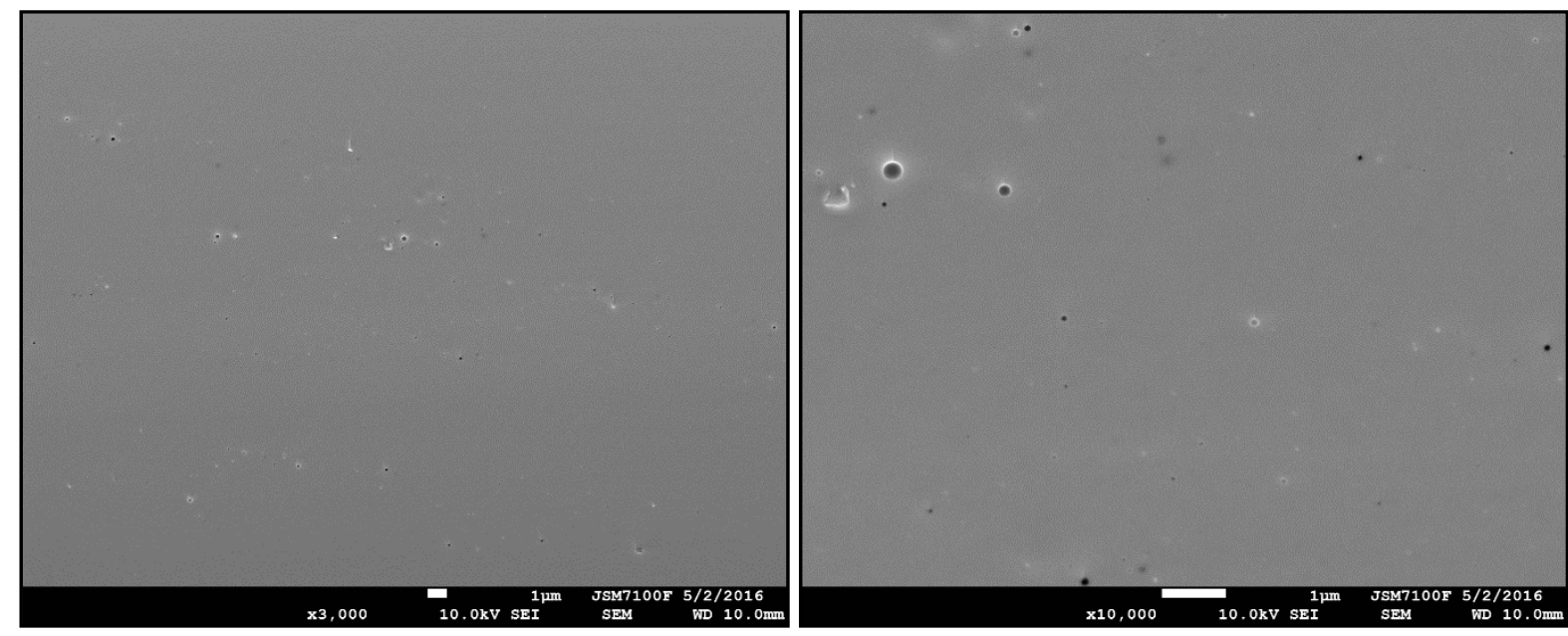

Figure 5. SEM images of the glass by sintering SPS using aluminum foil at $120^{\circ} \mathrm{C}$ for 10 minutes obtained from TAS-G30 powder.

The images show dense areas perfectly homogeneous, while discontinuities are also present in the glasses under the form of large bubbles of about $300 \mu \mathrm{m}$ in diameter while the majority has an average diameter of $100 \mu \mathrm{m}$. These defects explain the lowering in transmission intensity between the samples obtained by SPS relative to the base glass. Two assumptions can be put forward to explain the presence of bubbles, the surface oxidation when prepared outside or the glass evaporation when sintered. This second parameter will detailed later.

Finally, the last tests were conducted on tungsten carbide molds (WC) to remove any possible contamination of the carbon by the graphite molds. The WC is chosen for its chemical inertness and high temperature stability.

Changing molds involves changes in sintering parameters to achieve densification and therefore enhanced transmission. 


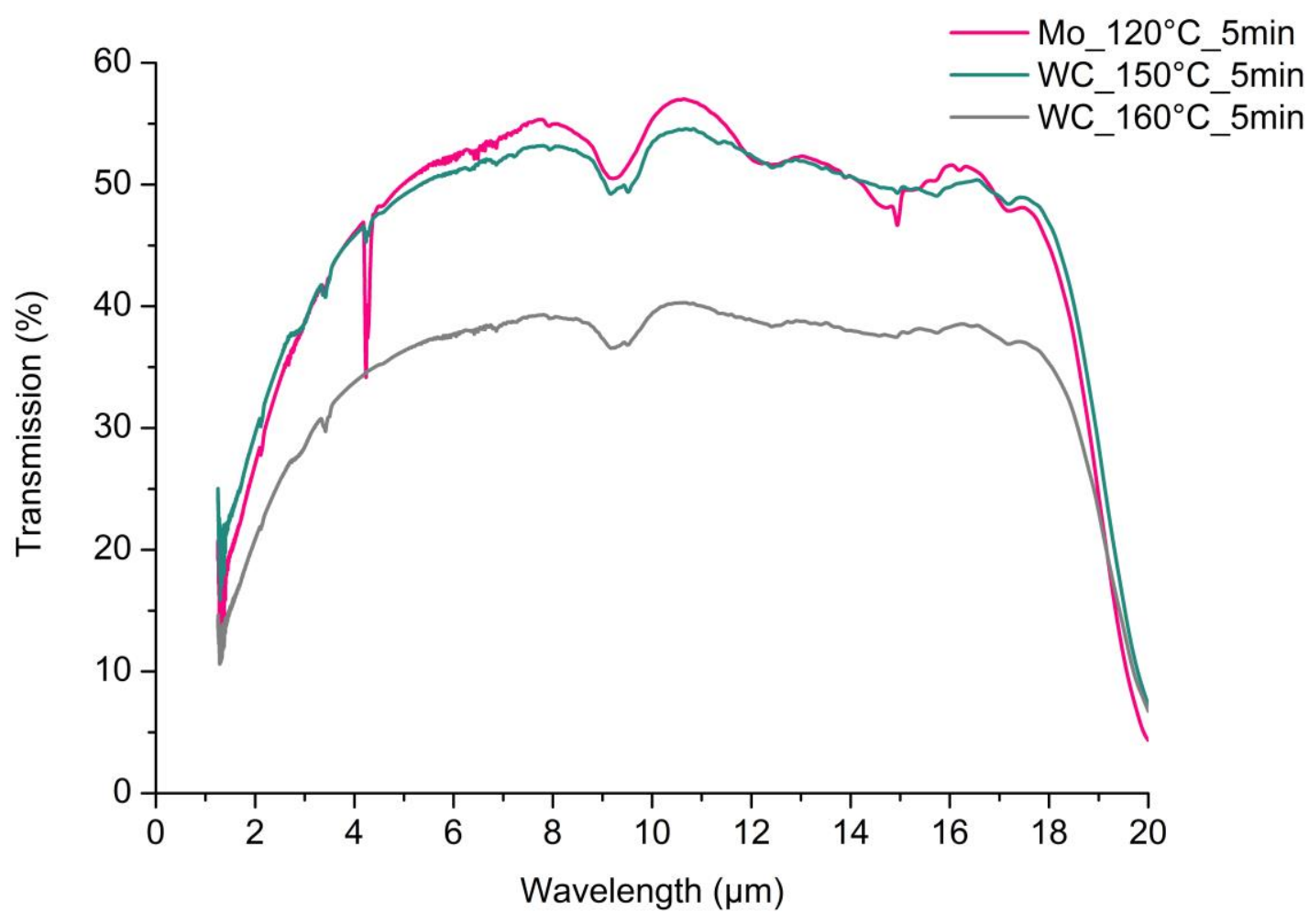

Figure 6. Transmission curves of glasses sintered in a WC mold starting from TAS-G30 powder.

Pellets obtained from TAS-G30 powder sintered in WC present close transmission window to that obtained previously by using a graphite mold with a molybdenum sheet.

It is therefore deduced that the transmission loss obtained on the sintered pellets in graphite molds using a molybdenum or aluminum foil, are not due to the diffusion of carbon in the samples but only to bubbles Thus, we can conclude the effectiveness of molybdenum and aluminum as an efficient barrier to the diffusion of carbon during SPS sintering.

One drawback to the use of WC molds is the need to apply higher temperature of sintering which could lead to a non-controlled crystallization of the sample therefore diminishing its optical properties. At $160^{\circ} \mathrm{C}$, scatterings occur due to fast crystallization of the samples. As seen previously, the milled powder is susceptible to crystallize, so further experiments were performed using graphite molds.

Once the parameters of temperature, pressure and dwell time are determined, new sintering are carried out on green samples obtained from powder milled for 160 hours, TAS-MM160. Transmission curves of obtained samples are recorded in Figure 7. 


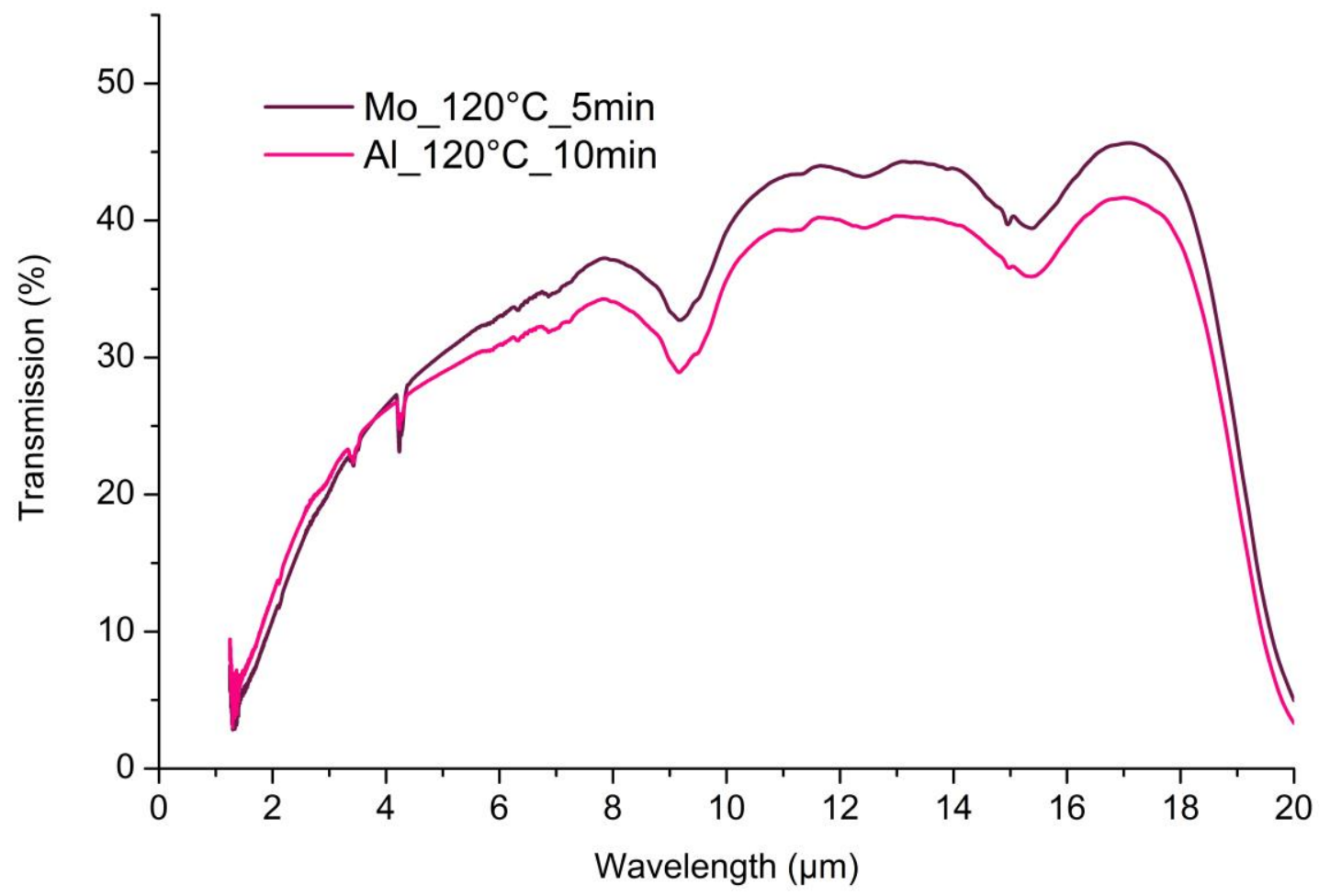

Figure 7. Transmission of bulk samples obtained by SPS sintering of TAS milled powder for 160h.

It may be noted that at similar temperatures and times to those used on the TAS-G30 powder for 30 minutes, while the window of transparency is still ranging from 2 to $20 \mu \mathrm{m}$, the obtained transmissions are lower, $47 \%$ instead of $57 \%$. Table 2 presents the compactness and density of obtained samples.

Table 2. Characteristics of bulk materials obtained by sintering the TAS-MM160 powders.

\begin{tabular}{c|ccc}
\hline & Thickness (mm) & Density & Densification (\%) \\
& & $\left( \pm 0,01 \mathrm{~g} . \mathrm{cm}^{-3}\right)$ & \\
\hline Mo_120C_5min & 1.40 & 4.80 & 98.4 \\
Al_120C_10min & 1.48 & 4.81 & 98.6 \\
\hline
\end{tabular}

Indeed, density measurements also show slightly lower densification to that obtained previously. SEM images (Figure 8) performed on the pellet sintered at $110^{\circ} \mathrm{C}$ for 10 minutes using a molybdenum foil confirm the remarks. In fact, the number of bubbles is increased throughout the sample and present bigger diameter ranging from 300 to 800 microns. The high residual porosity induces significant Rayleigh and MIE scatterings of light inducing a loss of transmission. 

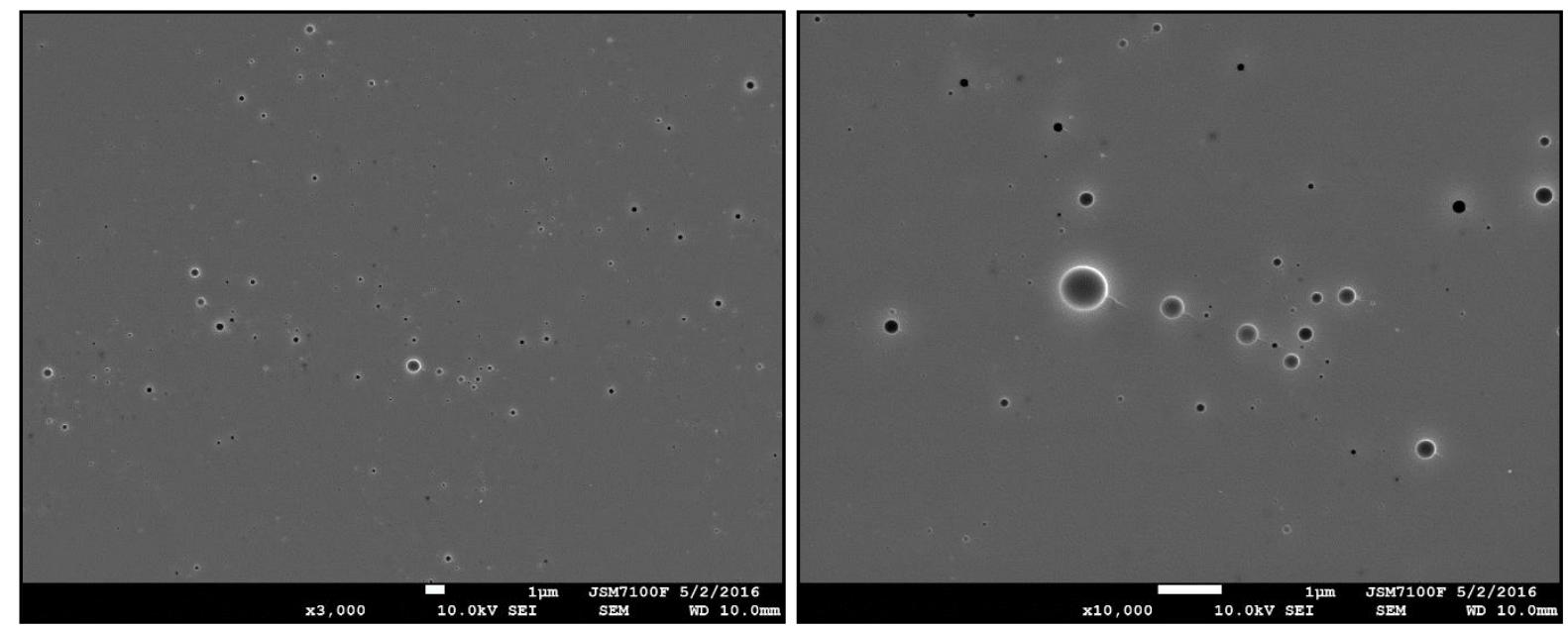

Figure 8.SEM images of the glass obtained by SPS sintering using a molybdenum foil at $110^{\circ} \mathrm{C}$ for 10 minutes obtained from TAS-MM160.

The presence of bubbles may be explained by the fact that the powders obtained after 160 hours of grinding have a lower size than the glass milled for 30 minutes. Two assumptions can explain this phenomenon. First, the decrease of powder with increase milling time leads to a higher specific surface and thus a higher oxidation. The remaining close pores after pre-pelletizing lead to irreversible bubbles. As already mentioned, the second possibility is the glass deterioration during sintering The EDS analysis performed confirms this hypothesis, revealing atomic percentages of $44 \%$ of selenium in the sintered pellet while this percentage is of $50 \%$ in the base powder.

Thus experiments were then performed under argon to minimize Se losses under vacuum. However higher temperatures have to be reached to enhance the transmission when using argon which could lead to crystallization (figure 9) 


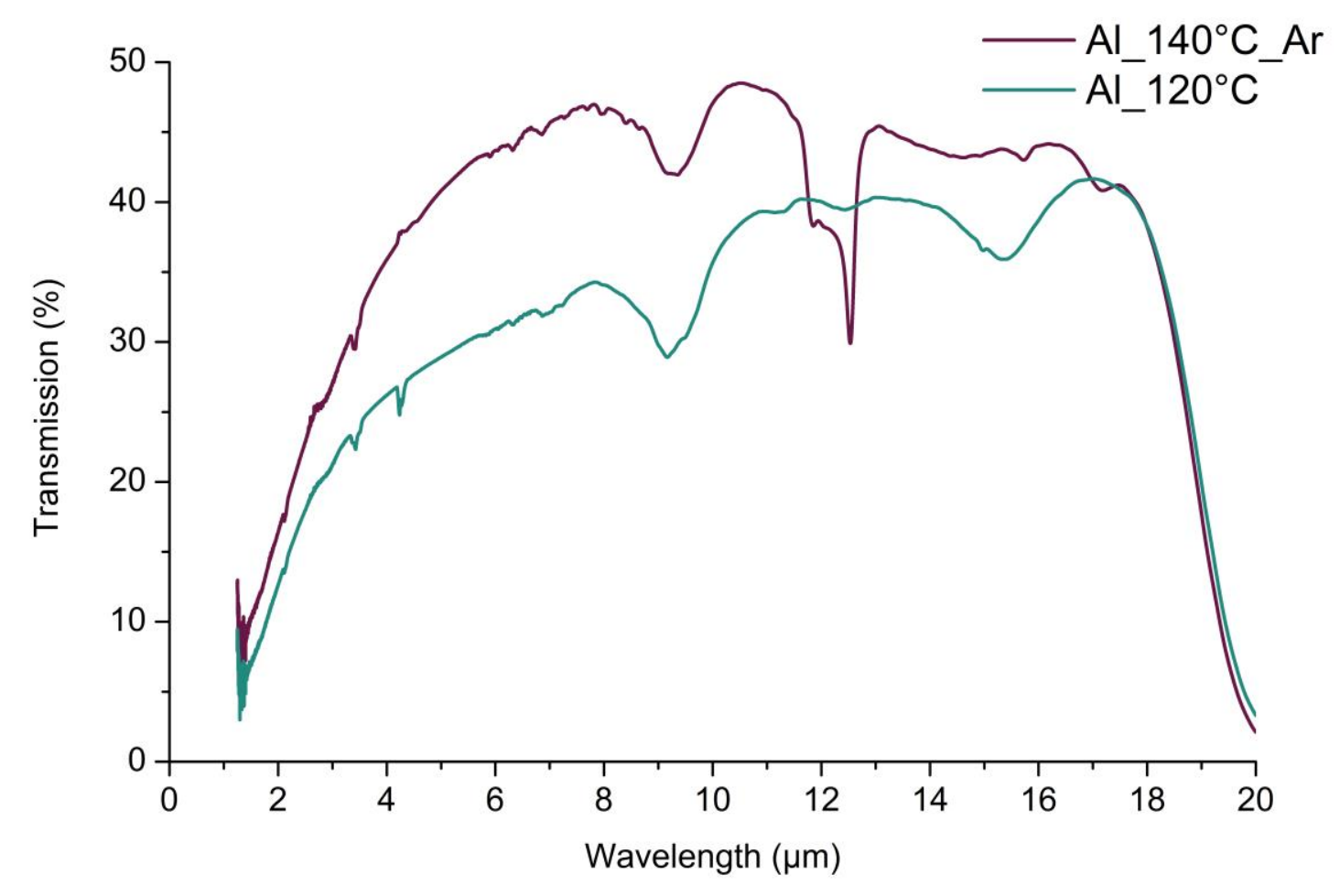

Figure 9: Transmission of bulk glasses obtained from sintering of the TAS-MM160 powder at high temperature.

SEM images made on a slice of TAS obtained by sintering at $140^{\circ} \mathrm{C}$ by milled powder show oval bubbles demonstrating that a lower viscosity is reached facilitating the glass shaping.

The transmission obtained on a pellet of TAS-MM160 sintered at $140^{\circ} \mathrm{C}$ for 5 minutes under argon lead to an increase of the maximum of transmission in the shorter wavelengths (figure 9). It can be assumed that sintering under argon reduces selenium evaporation, discontinuities in the massif, which thus decreases the scatterings and increases the maximum of transmission.

The Vickers hardness was then measured on sintered samples in order to compare with glasses made in silica tubes. TAS-MM-160h samples sintered at $140^{\circ} \mathrm{C}$ for $5 \mathrm{~min}$ present a hardness of $124.9 \pm 2 \mathrm{Hv}$ while the base glass synthesized in silica tubes has a hardness of $120.1 \pm 3 \mathrm{Hv}$. This slight increase can be explained by the strong compression undergone by the sintered pellets which was not annealed close to Tg to lower inner constrains.

\section{Structural study}

Structural study of mechanical milled powder, sintered glasses and TAS base glass were compared using Raman spectroscopy. In the figure 10 are presented the Raman spectra of mechanical milled powder after $100 \mathrm{~h}$ and $160 \mathrm{~h}$, of a TAS sintered pellet TAS-MM160-140 ${ }^{\circ} \mathrm{C}-5 \mathrm{~min}$ and tellurium. 


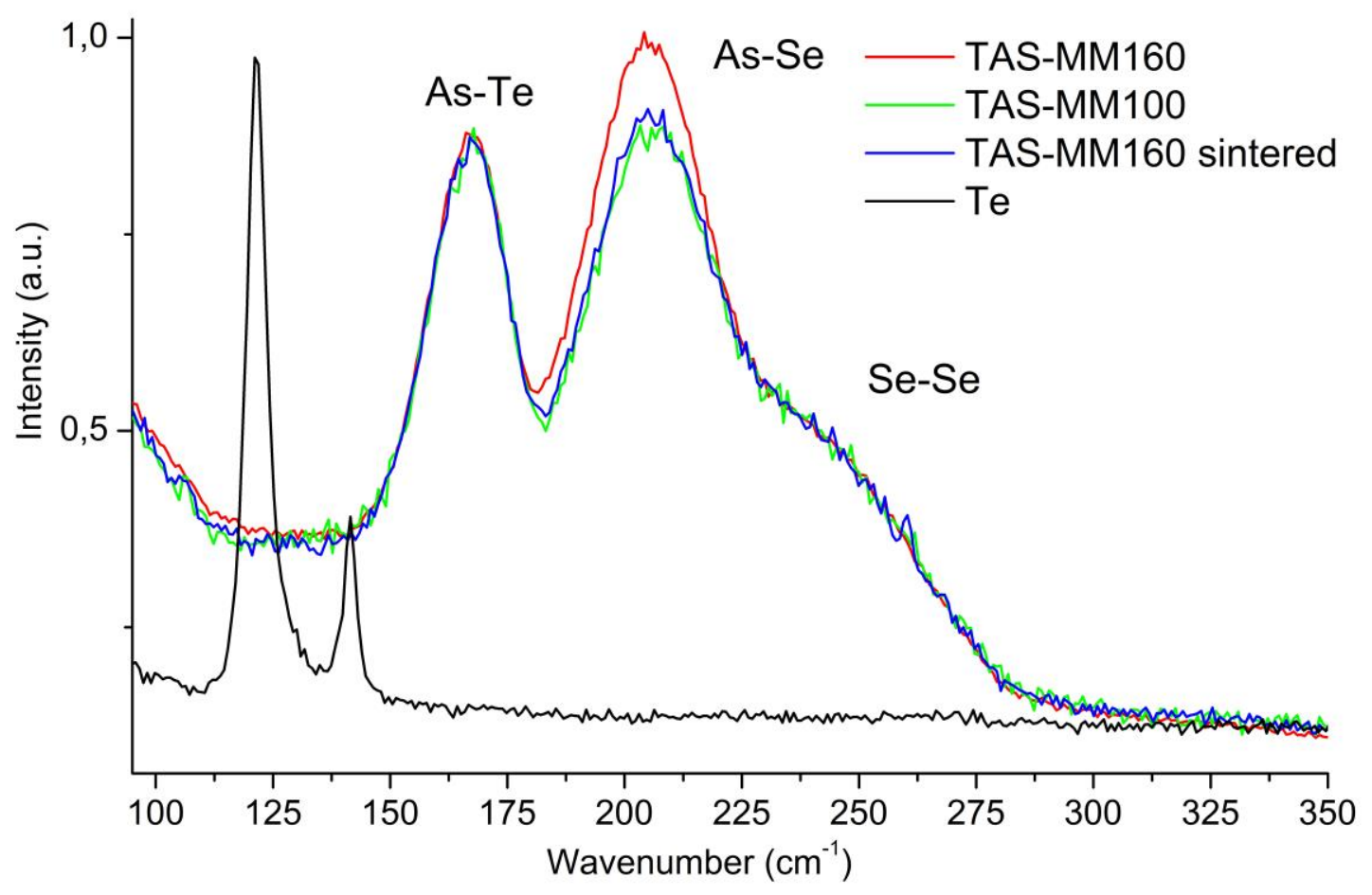

Figure 10. Raman spectra of mechanical milled powder after $100 \mathrm{~h}$ and $160 \mathrm{~h}$, of a TAS SPS sintered pellet TAS-MM160 at $140^{\circ} \mathrm{C}$ for $5 \mathrm{~min}$ and pure tellurium.

According to [13], the TAS-MM100 powder shows an intensity ratio of the different peaks corresponding to a richer tellurium glass than the usual $\mathrm{Te}_{20} \mathrm{As}_{30} \mathrm{Se}_{50}$ with a composition close to the $\mathrm{Te}_{20} \mathrm{As}_{40} \mathrm{Se}_{40}$. It corroborates the XRD results which showed a longer process of integration of $\mathrm{Te}$ within the glassy network.

If we refer to Jovari et al. [14], our experimental data shows the absence of Te-Te bonds demonstrating homogeneous distribution of tellurium in the glassy system. Indeed, tellurium introduced into the vitreous system $\mathrm{As}_{30} \mathrm{Se}_{70}$ destroyed Se-Se bonds, one can notice the absence of a peak at $250-270 \mathrm{~cm}^{-1}$. However, the shoulder at $238 \mathrm{~cm}^{-1}$ corresponds to selenium chains. As indicated by G. Delaizir et al [15], two peaks identified at 168 and $205 \mathrm{~cm}^{-1}$ correspond to the stretching of As-Te bonds and As-Se vibration. Moreover according to this paper, raman spectra of the TAS-MM160 and of the TAS base glass are identical demonstrating that the final powder (TASMM160h) has a very close structure as glass synthesized by melting / quenching.

A decrease of As-Se/As-Te bonds ratio is observed in the sintered TAS-MM160 compared to the TAS-MM160 powder which is confirmed by EDS analysis showing a As/Te ratio of 1.2 to 1.5 respectively. A loss of As-Se by evaporation when sintered under vacuum can be assumed.

MAS NMR spectra of the 77Se were recorded on the powder collected after 2, 5, 10, 15, 100 and 160 hours of milling as well as the $\mathrm{As}_{30} \mathrm{Se}_{70}$ glass (Figure 11). According to B. Bureau [16-17] and for a better understanding of the NMR spectra, lines corresponding to the three expected types of Se coordination, Se- Se- Se, Se- Se- As at and As-Se -As, were added respectively at 820ppm, 550 ppm, 
$360 \mathrm{ppm}$. In order to understand the incorporation effect of $\mathrm{Te}$, the $\mathrm{As}_{30} \mathrm{Se}_{70}$ composition has been selected as it corresponds to theTe ${ }_{20} \mathrm{As}_{30} \mathrm{Se}_{50}$ with a substitution of $20 \%$ of tellurium by selenium.

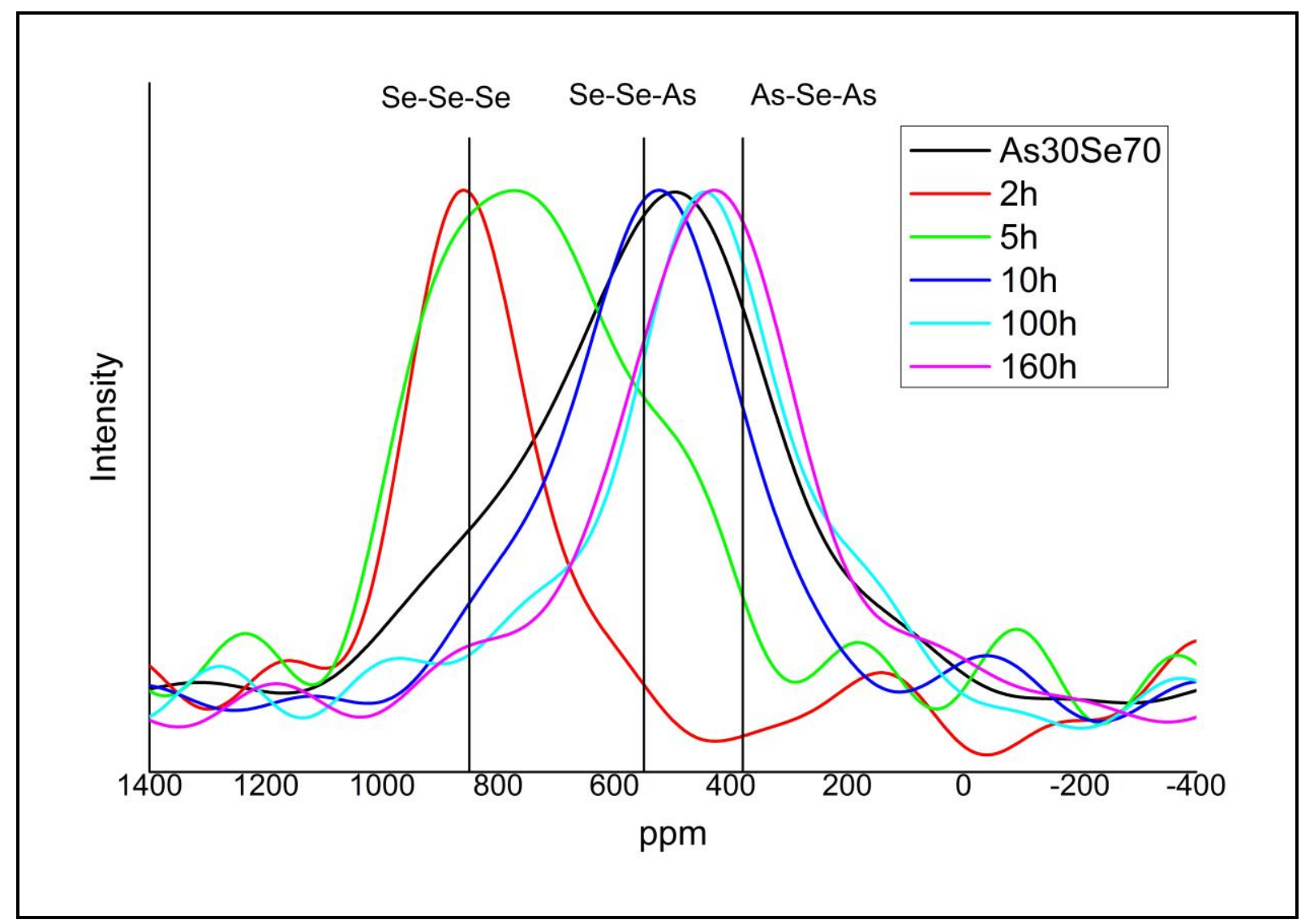

Figure 11: NMR spectra (MAS) of ${ }^{77}$ Se of powder obtained after 2, 5, 10, 15, 100 and 160 of milling and the $\mathrm{As}_{30} \mathrm{Se}_{70}$ base glass.

In Figure 11, we see that after only 2 hours of milling, selenium is amorphized probably forming selenium chains. With increased milling time, selenium starts to react with arsenic since after 5 hours of milling two environments are observed between 550 and $820 \mathrm{ppm}$, forming $\mathrm{AsSe}_{3}$ pyramids connected by Se bonds.

After 10 hours of milling the powder structure is very close to the reference $\mathrm{As}_{30} \mathrm{Se}_{70}$ glass without tellurium. This result is consistent with the XRD diffractograms demonstrating that Te enters later in the glassy network.

When mechanical alloying exceeds 10 hours, there are no longer any significant changes in the structure of milled powder from the viewpoint of selenium. Tellurium is incorporated into the glassy matrix without changing the shape of the NMR spectrum. Tellurium and selenium are in the same column of the periodic table, tellurium therefore preferentially substitutes for selenium rather than arsenic in the vitreous system. As already demonstrated in previous work, the substitution of selenium by tellurium induces a global right-shift of the entire spectrum of about 100ppm, leading to the disappearance of the Se-Se-Se bonds [18].

In addition, the spectrum of the milled powder 160 hours shows no difference with that obtained for the glass synthesized by melting / quenching [15]. The two curves show the same form attesting same structure regardless of the method of synthesis, mechanical milling or melt quenching.

\section{Conclusion}


Mechanical milling of metalloids elements $\mathrm{Te}$, As, Se permit to achieve $\mathrm{Te}_{20} \mathrm{As} 30 \mathrm{Se}_{50}$ amorphous powder without using silica tubes sealed under vacuum. Amorphization process was followed by various analytical techniques such as X-ray diffraction, differential scanning calorimetry, Raman spectroscopy, and NMR. It was observed that the selenium becomes quickly amorphous progressively reacting with arsenic whereas the tellurium and arsenic require more time to be incorporated into the final vitreous phase. After 40 hours of mechanical alloying, the obtained powder while presenting an identical $\mathrm{Tg}\left(135^{\circ} \mathrm{C}\right)$ than the reference glass, a crystallization peak is still observable demonstrating a structural deviation. 160 hours are mandatory to reach the thermal answer in DSC curves that is to say without crystallization peak. Indeed, mechanical milling allows reaching a metastable state of the TAS glass that is nevertheless known to be a stable glass. This metastable state opens the way to produce transparent glass-ceramics as already demonstrated in previous papers with different chalcogenide glasses [19-20].

The different analytical methods, Raman spectroscopy, NMR experiments, were also used to compare the structures of amorphous powders made by mechanical milling with the structure of the glass synthesized by melt-quenching. The results collected have confirmed that this process is efficient to achieve the same glass structure.

Different parameters of sintering were studied to produce bulk glasses from milled powders. Very short times combined to sintering temperatures close to the glass $\mathrm{Tg}$ is required to produce solid materials having a density and good optical properties. Protection foils of aluminum and molybdenum used between the graphite mold and the sample limit the diffusion of carbon within the glassy matrix and the scatterings as well.

Furthermore, the transmission curves of sintered samples showed the presence of absorption bands, due to oxygen or hydrogen. These impurities may originate from the starting components, the grinding step or the sintering step. In fact, even if the elements constituting the test compounds were stored and milled under a controlled argon atmosphere, powder is taken out of the glove box before sintering. The high specific surface of the powders obtained by mechanical alloying causes rapid surface oxidation in air which is dendrimental when sintered, leading to the formation of bubbles. Given the encouraging results and the possibility to optimize them, next steps will consist in molding and sintering sensors in a special designed mold.

\section{References}

1. X.H. Zhang, Y. Guimond, Y. Bellec, J. Non-Cryst. Solids 326\&327 (2003) 519-523.

2. E. Guillevic, X.H. Zhang, T. Pain, L. Calvez, J.L Adam, J. Lucas, S. Ollivier, M. Guilloux-Viry, G. Gadret, Opt. Mater. 31 (11) (2009) 1688-1692.

3. M-L Anne, J. Keirsse, V. Nazabal, S. Inoue, C. Boussard-Pledel, J. Charrier, K. Yanakata, O. Loreal, J. Le Person, C. Compère, B. Bureau, Sensors 9 (2009) 7398-7411.

4. V.S. Shiryaev, J.L. Adam, X.H. Zhang, C. Boussard-Pledel, J. Lucas, M.F. Churbanov, 2004, J. Non-Cryst. Solids 336 (2004) 113-119.

5. G. S. Athanasiou, J. Ernst, D. Furniss, T. M. Benson, J. Chauhan, J. Middleton, C. Parmenter, M. Fay, N. Neate, V. Shiryaev, M. F. Churbanov, A. B. Seddon, J. Lightwave Technol. 34(4) (2016) 1212-1219.

6. C. Koch, O.B. CavinB, C.G. McKamey, J.O. Scarbrough, Appl. Phys. Lett. 43 (1984) 1017-1019

7. Y.H. Ji, Z.H. Ge, Z. Li, J.Feng, J. Alloys Comp. 680 (2016) 273-277.

8. M. Hubert, G. Delaizir, J. Monnier, C. Godart., H.L Ma, X.H Zhang, L. Calvez, Opt. Express. 19(23) (2011) 23513.

9. E. Petracovschi, M. Hubert, J.L. Adam, X.H. Zhang, L. Calvez, Phys.Solid State-b, 251(7) (2014) $1330-1333$.

10. X.H. Zhang, H.L. Ma, C. Blanchetière, J. Lucas, J. Non-Cryst. Solids, 161 (1993) 327 
11. D.Le Coq, C. Boussard-Pledel, G. Fonteneau, T. Pain, B. Bureau, J.L. Adam, J. Non-Cryst. Solids $326 \& 327$ (2003) 451-454.

12. A. Bertrand, J. Carreaud, G. Delaizir, J. Duclere, M. Colas, J. Cornette, M. Vandenhende, V. Couderc, P. Thomas, J. Am. Ceram. Soc. 97 (1) (2013) 163-172.

13. Structural investigation of chalcogenide and chalcohalide glasses using Raman spectroscopy. Nguyen V-Q, Sanghera J-S, Freitas J-A, Aggarwal I-D, Lloyd I-K., J. Non-Cryst. Solids 248 (1999) 103-114.

14. P. Jóvári, B. Bureau, I. Kabanc, V. Nazabal, B. Beuneu, U. Rütt, J. Alloys Comp. 488 (1-20) (2009) 39-43.

15. Structural characterizations of As-Se-Te glasses. Delaizir G, Dussauze M, Nazabal V, Lecante P, Dollé M, Rozier P, Kamitsos E-I, Jovari P, Bureau B., J. Alloys Comp. 509 (2011) 831-836.

16. B. Bureau, J. Troles, M. Le Floch, F. Smektala, G. Silly, J. Lucas, Solid State Sci. 5 (2003) 219224.

17. B. Bureau, J. Troles, M. Le Floch, F. Smektala, J. Lucas, J. Non-Cryst. Solids 326 (2003) 58-63.

18. B. Bureau, C. Boussard-Pledel, M. LeFloch, J. Troles, F. Smektala, J. Lucas, J. Phys.Chem. B. 109(13) (2005) 6130-6135

19. H.L. Ma, L.Calvez, B. Bureau, M. Le Floch, X.H. Zhang, J. Lucas, J. Phys. Chem. Solids, 68 (56) (2007) $968-971$

20. L. Calvez, H.L. Ma, J. Lucas, X.H. Zhang, J. Non-Cryst. Solids 354(12-13) (2008) 1123-1127. 